\title{
Die Informationstechnologien sind Sache der Privatwirtschaft
}

\section{René Buholzer}

\section{(2) OpenEdition \\ Journals}

Electronic version

URL: http://journals.openedition.org/sjep/547

DOI: $10.4000 /$ sjep.547

ISSN: 1663-9677

\section{Publisher}

Institut de hautes études internationales et du développement

\section{Printed version}

Date of publication: 1 novembre 2003

Number of pages: 133-134

ISSN: $1660-5926$

\section{Electronic reference}

René Buholzer, «Die Informationstechnologien sind Sache der Privatwirtschaft », Schweizerisches Jahrbuch für Entwicklungspolitik [Online], 22-2 | 2003, Online erschienen am: 10 Juni 2010, abgerufen am 08 September 2020. URL : http://journals.openedition.org/sjep/547 ; DOI : https://doi.org/ 10.4000/sjep.547 


\title{
Die Informationstechnologien sind Sache der Privatwirtschaft
}

\author{
René Buholzer*
}

W ir befinden uns im Übergang vom Industriezeitalter zur Informationsgesellschaft. Information, Wissen und Kommunikation erhalten eine immer grössere Bedeutung im Leben der einzelnen Menschen, wie auch im Leben von Firmen und Staaten. Daniel Bell hat dies bereits 1979 treffend analysiert: „The crucial point about a post-industrial society is that knowledge and information become the strategic and transforming resources of the society, just as capital and labour have been the strategic and transforming resources of the industrial society."

Basis dieser Entwicklung bilden die neuen Informations- und Kommunikationstechnologien (IKT). Wie mittlerweile wissenschaftlich vielfach belegt, spielen sie aus volkswirtschaftlicher Sicht eine Schlüsselrolle. Denn im Unterschied zu anderen technischen Innovationen betreffen Einsatz und kommerzielle Nutzung der IKT nicht nur einzelne, sondern nahezu alle Wirtschaftszweige. Sie sind eigentliche Basistechnologien. Auf makroökonomischer Ebene bewirken sie wichtige Wachstumsund Beschäftigungseffekte. Auf mikroökonomischer Ebene resultieren grundlegende Veränderungen.
Durch die Senkung der Informationsund Kommunikationskosten werden unternehmensintern wie -extern (Arbeitsteilung) ganze Wertschöpfungsketten weltweit neu organisiert.

Die wirtschaftshistorische Erfahrung zeigt, dass die Diffusion von Basistechnologien ein Prozess ist, der sehr lange dauert und teilweise unvorhergesehene Ergebnisse zeitigt. So führt etwa die weltweite Einführung der Internet-Technologie dazu, dass Information und Wissen nicht mehr ein Privileg von gewissen Gesellschaftsschichten - vornehmlich in reichen Industrieländern - ist, sondern potenziell in der ganzen Welt und fast allen gesellschaftlichen Gruppen zeitgleich zur Verfügung steht. Die Konsequenzen dieser Entwicklung können heute erst erahnt werden. Ein wesentlicher Grund für die lange Dauer dieses Diffusionsprozesses liegt darin, dass sich das volle Potenzial nur entfalten kann, wenn die zu den Investitionen in Basistechnologien erforderlichen komplementären Voraussetzungen geschaffen werden. Das heisst, dass die Veränderungen auf makround mikroökonomischer Ebene nur realisierbar sind, wenn eine Volkswirtschaft die notwendigen Rahmenbedingungen und das erforderliche Humankapital bereitstellt.

* Mitglied der Geschäftsleitung von economiesuisse. Er vertritt die Wirtschaft in der offiziellen Schweizer Delegation am WSIS. 
Der Wandel hin zu einer Wissensgesellschaft ist also weit mehr als die Bereitstellung der nötigen Infrastruktur für die neuen Technologien. Diese sind nur eine notwendige Voraussetzung. Man kann es mit der Erfindung des Buchdruckes vergleichen: Sie erlaubte die Demokratisierung des Wissens; doch die Menschen mussten zuerst lesen lernen. Der Weg zur Wissensgesellschaft kann deshalb nur gemeinsam gegangen werden. Der Staat, die Unternehmen und die Organisationen der Zivilgesellschaft müssen im Rahmen ihrer Kompetenzen und Fähigkeiten ihren Teil zum Gelingen beitragen.

Die Ursachen der Armut - wie der fehlende Zugang zu Energie, Wasser und Bildung - standen am Weltgipfel für nachhaltige Entwicklung im Zentrum (Johannesburg 2002). Dabei hat der Gipfel klargemacht, dass öffentliche Entwicklungshilfe allein die Armut nicht wirksam genug bekämpfen kann. Dazu braucht es vielmehr weltweiten Handel und Direktinvestitionen von Unternehmen. Diese sind aber ihrerseits auf gute Rahmenbedingungen, offene Märkte und Good Governance - wie etwa die Einhaltung der Menschenrechte - angewiesen.

Analoges gilt auch für die Herausforderungen zur Realisierung einer weltweiten Informationsgesellschaft. Staatliche Programme, neue Regulierungen oder Steuern auf IKT werden den digitalen Graben zwischen Nord und Süd nicht zuschütten können. Ohne den Beitrag der Wirtschaft kann diese grosse Herausforderung nicht bewältigt werden. Die nationale und internationale Politik ist deshalb gefordert, im Rahmen eines marktwirtschaftlichen Konzeptes ein innovations- und investitionsfreundliches
Klima für (KMU-)Unternehmen zu schaffen, wettbewerbsfreundliche Rahmenbedingungen für die Telekommunikation als Rückgrat der Informationsgesellschaft $\mathrm{zu}$ garantieren und die Benutzerbefähigung der breiten Bevölkerung durch Ausbildung zu fördern. Ferner kann die öffentliche Hand als Anwender (E-Government) und Impulsgeber nicht nur einen wichtigen Beitrag zur Bewusstseinsbildung und Akzeptanz der IKT leisten, sondern auch durch Reformen in der eignen Verwaltung ihre Effizienz (Steuererhebung, Bewilligungen, etc.) und Bürgernähe steigern.

Träger der IKT ist aber letztlich die Privatwirtschaft. Sie hat die Aufgabe, die IKT als Basistechnologie innovativ zu nutzen und sinnvoll einzusetzen. Sie hat aber auch die Verantwortung, mit diesen Möglichkeiten verantwortungsvoll umzugehen. Risiken bestehen. Etwa der Schutz der Privatsphäre und die Sicherheit. Hier ist die Wirtschaft gemeinsam mit dem Staat und der Zivilgesellschaft aufgerufen, Massnahmen zu ergreifen, diese Risiken zu minimieren, ohne den Nutzen dieser Technologien unnötig zu schmälern. Angesichts des enormen Potenzials der IKT als Instrument zum Wissensausbau, zur Steigerung der persönlichen Kompetenz und der politischen Willensbildung sollten deshalb die Themen Datenschutz und Datensicherheit positiv angegangen werden. Denn: Erkki Liikanen ist zuzustimmen, als er vor einigen Jahren festgestellt hat: „The alternative for business is simple: embrace digital technologies or die. This applies to society as a whole. If we do not change now, we will be left behind and lose at all levels: growth, employment, social cohesion." 